\title{
Orientation fluctuations in magnetotactic swimming
}

\author{
Sebastian Smyk ${ }^{1}$, Vitali Telezki ${ }^{1}$, Josef Riepl ${ }^{1,2}$, Johannes Hayes ${ }^{1,2}$, and Stefan Klumpp ${ }^{1, a}$ \\ ${ }^{1}$ Institute for the Dynamics of Complex Systems, University of Göttingen, Friedrich-Hund-Platz 1, 37077 Göttingen, \\ Germany \\ 2 Faculty of Physics, University of Regensburg, Universitätsstraße 31, 93051 Regensburg, Germany
}

Received 12 May 2020 / Accepted 5 January 2021 / Published online 15 April 2021

(C) The Author(s) 2021

\begin{abstract}
The fluctuations experienced by magnetic microswimmers in a magnetic field often have nonthermal contributions, in particular in the case of biological swimmers such as magnetotactic bacteria. Here we study a model for an active self-propelled particle subject to correlated internal noise as a model for the active, non-thermal noise contribution and determine the effect of the correlation time on the diffusion of the swimmer and on its orientation in a magnetic field. A description in terms of an effective temperature is possible, but has limitations.
\end{abstract}

\section{Introduction}

A key concept of statistical physics is that the fluctuations of observables provide information about the properties of the underlying physical systems and processes. Fluctuations in thermodynamic equilibrium are related to response quantities and their time correlations are linked to near-equilibrium dynamics via the fluctuation-dissipation theorem [1]. In many settings, in particular in living systems, far-from-equilibrium situations are common, where processes are coupled to the flow of energy, matter and/or information. In these situations, violations of the fluctuation-dissipation theorem have been used to demonstrate the presence of noise of non-thermal origin and to separate thermal and nonthermal noise $[2,3]$. Another indicator of the presence of non-thermal noise is the need for an effective temperature rather than the actual temperature for the description of fluctuations. One example where effective temperatures have been used is given by the orientation fluctuations of natural magnetic microswimmers, magnetotactic bacteria [4-7].

Microswimmers, micron-scale particles that self-propel in fluids, have been studied extensively over the last years, from a view point of basic science as well as with respect to environmental and biomedical applications [8-10]. Magnetic microswimmers (natural, synthetic or biohybrid) are particularly promising, because their motion can be controlled remotely with a magnetic field [10]. Magnetotactic bacteria, which naturally form an intracellular chain of magnetic nanoparticles, the magnetosome chain, to align with magnetic fields and swim along the field lines, have been studied in particular [10-12]. Since their interaction energy with

${ }^{a}$ e-mail: stefan.klumpp@phys.uni-goettingen.de (corresponding author) typical magnetic fields (the Earth's magnetic field in their natural environment) is of the order of $10 k_{\mathrm{B}} T$, orientation fluctuations are common. They may also be important functionally, as they allow the bacteria to transiently deviate from the direction defined by the field, e.g. to circumvent obstacles in their path. Several studies of orientation fluctuations have shown that the magnitude of these fluctuations exceeds what is expected based on thermal fluctuations and have used effective temperatures to describe them [4-7]. Usually, the excess noise is ascribed to internal processes of the living organism, an interpretation supported by an early study that showed smaller orientation fluctuations for dead bacteria [5]. Here we consider a model for the combination of thermal and internal "active" noise, where the latter is described as colored noise and explore its consequences for the orientation fluctuations as well as the effective (active) diffusive motion of the swimmer.

\section{Model}

We consider a single active Brownian particle [9], which is described by its position $\mathbf{r}$ and orientation $\mathbf{e}=(\cos \varphi, \sin \varphi)$, both for simplicity taken in twodimensional space. The orientation defines the direction of self-propulsion with speed $v$ and is also the direction of the magnetic moment $\mathbf{m}=m \mathbf{e}$.

The equations of motion for an active Brownian particle with the position $\mathbf{r}$ and the orientation angle $\varphi$ in a homogeneous external magnetic field with strength $B$ are 


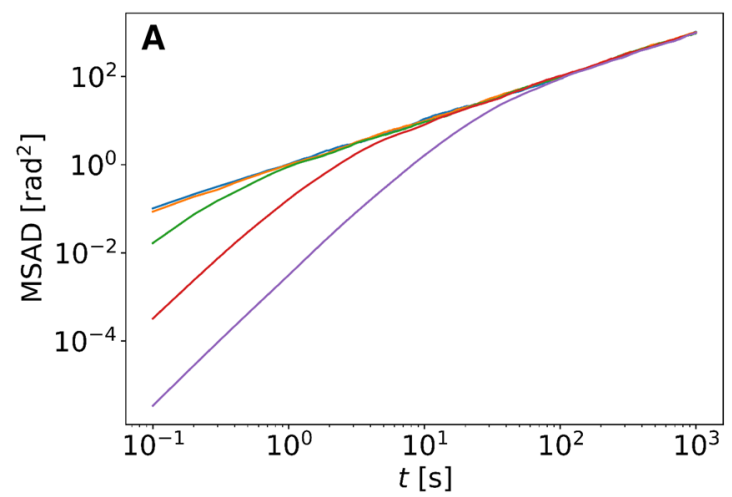

Fig. 1 a Mean square angular displacement (MSAD) and (b) mean square displacement (MSD) of the swimmers as functions of time for different correlation times $\tau$ (col-

$$
\begin{aligned}
& \dot{\mathbf{r}}=v \mathbf{e}+\sqrt{2 D_{t}} \boldsymbol{\xi} \\
& \dot{\varphi}=-\frac{1}{\gamma_{r}} m B \sin \varphi+\sqrt{2 D_{r}} \xi_{3}+\sqrt{\frac{D_{\mathrm{c}}}{\tau}} \eta_{c} .
\end{aligned}
$$

Here $\boldsymbol{\xi}=\left(\xi_{1}, \xi_{2}\right)$ and $\xi_{3}$ are thermal white noise with $\left\langle\xi_{i}(t)\right\rangle=0$ and $\left\langle\xi_{i}(t) \xi_{j}\left(t^{\prime}\right)\right\rangle=\delta_{i, j} \delta\left(t-t^{\prime}\right)$, where the indices $i$ and $j$ run over the components of the noise. Its noise strength is given by the translational and rotational diffusion coefficients $D_{t}$ and $D_{r} . \eta_{c}$ is the colored noise describing noise from internal non-thermal processes, characterized by a correlation time $\tau$, i.e. with

$$
\left\langle\eta_{c}(t) \eta_{c}\left(t^{\prime}\right)\right\rangle \propto \mathrm{e}^{-\left|t-t^{\prime}\right| / \tau} .
$$

The coefficient of the colored noise in Eq. 2 was chosen to obtain $\left\langle\varphi^{2}(t)\right\rangle=2 D_{\mathrm{c}} t$ in the long time limit for the case $D_{r}=0$. Colored noise $\eta_{c}$ can be generated from white noise $\xi_{4}$ via the additional equation of motion

$$
\dot{\eta}_{c}=-\frac{1}{\tau} \eta_{c}+\sqrt{\frac{2}{\tau}} \xi_{4}
$$

The coefficients were chosen so that the colored noise $\eta_{c}$ is Gaussian with $\left\langle\eta_{c}(t)\right\rangle=0$ and $\left\langle\eta_{c}^{2}(t)\right\rangle=1$ in the long time limit.

For the numerical solution, the equations of motion were integrated using the Euler-Maruyama method [13] with a time step of $10^{-5} \mathrm{~s}$. The statistical analysis was performed over 500 or 1000 realizations.

\section{Results and discussion}

We first consider the motion of the active particle in the absence of a magnetic field $(B=0)$ and without thermal noise $\left(D_{r}=D_{t}=0\right)$. In that case, the Langevin equation for the angle $\varphi$ can be solved analytically (in fact this equation is mathematically equivalent to the standard Langevin equation that is obtained by adding

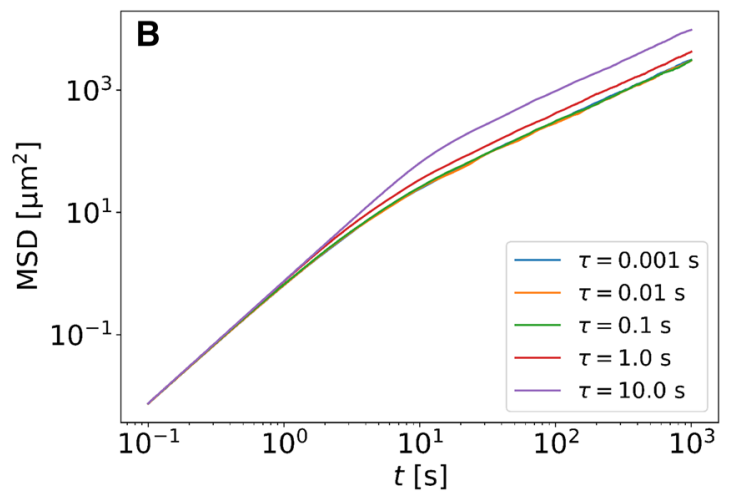

ored lines) and fixed $D_{\mathrm{c}}=0.5 \mathrm{rad}^{2} / \mathrm{s}, v=0.87 \mu \mathrm{m} / \mathrm{s}$, $D_{r}=D_{t}=0$. Statistical analysis was performed over 1000 realizations

noise to a Newtonian equation of motion with damping [14]).

Figure 1a shows the mean square angular displacement (MSAD) for different realizations of the same value of $D_{\text {c }}$ with different values of $\tau$ (colored lines). From the analytical solution, the MSAD is found to be $\left\langle\varphi^{2}\right\rangle \approx D_{\mathrm{c}} t^{2} / \tau$ for short times $t$ and $\left\langle\varphi^{2}\right\rangle \approx 2 D_{\mathrm{c}} t$ for long times. The long time behavior is independent of the correlation time and sets in after time $2 \tau$.

Moments of the position of the active particle are calculated by numerical integration. Figure $1 \mathrm{~b}$ shows the mean square displacement (MSD). The position initially exhibits ballistic motion, $\left\langle x^{2}\right\rangle=\left\langle y^{2}\right\rangle \approx v^{2} t^{2}$, and then crosses over to diffusive motion with $\left\langle x^{2}\right\rangle=\left\langle y^{2}\right\rangle \approx$ $2 D_{\text {eff }} t$ after a persistence time $2 \tau_{\mathrm{p}}$. The effective (translational) diffusion coefficient is given by $D_{\text {eff }}=D_{0}=$ $v^{2} / D_{\mathrm{c}}$ for short correlation times $\tau$, i.e. in the limit of white noise. In that case, $\tau_{\mathrm{p}} \approx 1 / D_{\mathrm{c}}$. The persistence time increases with increasing correlation time $\tau$ as shown in Fig. 1b. Figure 2 shows the dependence of the effective translational diffusion coefficient $D_{\text {eff }}$ on the correlation time $\tau$ for different values of $D_{\mathrm{c}}$ (colored points). Just like the persistence time, the effective diffusion coefficient increases with increasing correlation time. The slope in Fig. 2 indicates a dependence $D_{\text {eff }}-D_{0} \propto \tau^{1 / 2}$ (solid lines). The only relevant parameter with the dimension of length is the self-propulsion velocity. Therefore, the diffusion coefficient must be given by $D_{\text {eff }}=v^{2} \tau_{\mathrm{p}}$, where the required timescale can be identified with the persistence time. The latter can be decomposed as the white-noise persistence time and a $\tau$-dependent contribution as $\tau_{p}=1 / D_{\mathrm{c}}+\Delta \tau_{\mathrm{p}}(\tau)$. Since we numerically observe a $\tau^{1 / 2}$-dependence and $1 / D_{c}$ is the only other time scale in the model, we have $\Delta \tau_{\mathrm{p}}=a\left(\tau / D_{c}\right)^{1 / 2}$ with a numerical constant $a$. We note that the time scale in the correction term due to the noise correlations is given by the geometric mean of the two relevant time scales related to a change in orientation, the time scale of rotational diffusion $1 / D_{\mathrm{c}}$ and the correlation time of the noise $\tau$ that generates the rotational diffusion. The geometric mean is known to provide a single characteristic time scale in decay 


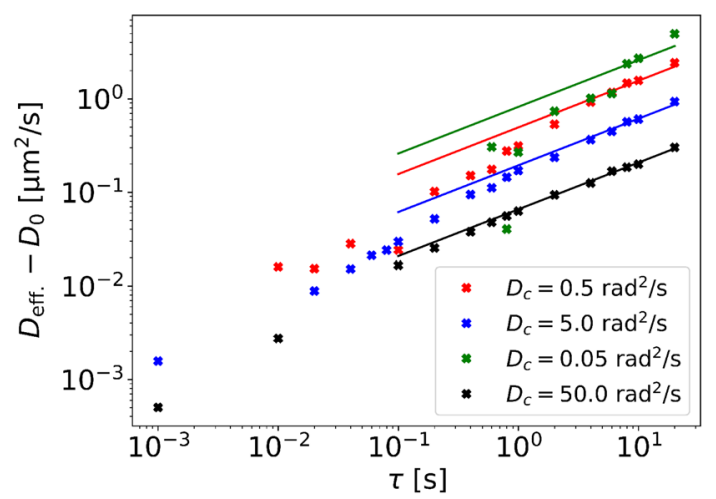

Fig. 2 Effect of the correlation time $\tau$ of the colored noise on the effective translational diffusion coefficient $D_{\text {eff }}$ for different values of $D_{\mathrm{c}}$. For each $D_{\mathrm{c}}$ the numerical constant $a$ in Eq. 2 was determined via non-linear least squares regression (lines)

processes [15]. Furthermore, the factor $\left(\tau D_{\mathrm{c}}\right)^{1 / 2}$ is also known as the expected angular change during the correlation time $\tau$ [16]. Overall, the numerical observation and dimensional considerations together suggest the relation

$$
D_{\text {eff }} \simeq D_{0}+a v^{2}\left(\tau / D_{\mathrm{c}}\right)^{1 / 2}=\frac{v^{2}}{D_{\mathrm{c}}} \times\left[1+a\left(\tau D_{\mathrm{c}}\right)^{1 / 2}\right]
$$

for the effective diffusion coefficient.

Next we consider the orientation of the swimmer in an external homogeneous magnetic field. We vary the field strength $B$ in the numerical integration and plot $\langle\cos \varphi\rangle$, the average of the projection of the magnetic moment on the direction of the field in Fig. 3a. This quantity usually follows a Langevin function and depends on $m B / k_{\mathrm{B}} T$ [17]. In our case the noise is not thermal. Yet, the qualitative behavior still follows the Langevin function, however with an effective temperature $T_{\text {eff }}$. This effective temperature can be determined from the field strength $B^{*}$ for which $\langle\cos \varphi\rangle=0.5$, which we obtain via linear interpolation from our numerical data. The Langevin function takes the value 0.5 for $m B^{*} /\left(k_{\mathrm{B}} T_{\text {eff }}\right) \simeq 1.79$, from which we determine the effective temperature. As expected the effective temperature increases as a function of the noise strength $D_{\mathrm{c}}$, but is seen to decrease as a function of the correlation time $\tau$, as shown in Fig. 3b. A decrease as a function of $\tau$ agrees with the results obtained from a small- $\tau$ expansion for the diffusion coefficient in the corresponding Fokker-Planck equation [16]. However, one has to be careful here: The effective temperature that is obtained from the Langevin function (and thus from the fluctuations around an equilibrium in the field) does not describe the free diffusion of the angular variable as measured by the mean square angular displacement in the absence of a field. In fact, one can define an alternative effective temperature from the latter as $k_{\mathrm{B}} T_{\mathrm{eff}}^{(\mathrm{r})}=D_{\mathrm{c}} \gamma_{\mathrm{r}}$. In contrast to the temperature obtained from the Langevin curve, this temperature is independent of the correlation time, as illustrated in Fig. 3b. Moreover, its value does not agree with $T_{\text {eff }}$ even in the limit $\tau \longrightarrow 0$ as the Stokes-Einstein relation is in general not fulfilled. Likewise, though probably of less use, one could define yet another temperature from the effective translational diffusion, $k_{\mathrm{B}} T_{\text {eff }}^{(\mathrm{t})}=D_{\text {eff }} \gamma_{\mathrm{t}}$, which depends on the self-propulsion velocity and is seen to increase as a function of $\tau$. We note that the three effective temperatures are controlled by different parameters: $T_{\text {eff }}$ depends on $D_{\text {c }}$ and on the correlation time of the colored noise, while $T_{\mathrm{eff}}^{(\mathrm{r})}$ is controlled by $D_{\mathrm{c}}$ alone. Neither of the two depends on the self-propulsion velocity. In contrast, $T_{\text {eff }}^{(\mathrm{t})}$ depends on the self-propulsion velocity as well as the colored noise, which determines the persistence time of the active motion. These different dependencies indicate that the concept of an effective temperature is only of limited use.

Finally, we briefly consider the case with both thermal noise and correlated non-thermal noise and plot again a Langevin curve for the orientation in a magnetic field, as this is the quantity of experimental interest, as well as the corresponding effective temperature. The results are shown in Fig. 4. Not surprisingly, stronger fields are needed for the same degree of alignment in the case of thermal and correlated noise compared to thermal noise only (black line). The maximal shift of the Langevin curve is obtained in the limit of white noise. This observation provides a lower limit on the required strength of the non-thermal noise as quantified by $D_{\mathrm{c}}$. Experimentally observed effective temperatures exceed the actual temperature by a factor in the range $1-10$ [4-6]. To obtain such an increase in effective temperature, sufficiently large $D_{\mathrm{c}}$ are required, with larger $D_{\mathrm{c}}$ required for longer correlation times. With the parameters used in Fig. 4, an effective temperature comparable to experimentally observed ones is found for realistically small (sub-second) correlation times. However, to determine both strength and correlation time of the nonthermal noise the Langevin curve that is determined in experiments alone is not sufficient, rather a direct measurement of the free rotational diffusion would be needed in addition.

In conclusion, we have studied the effect of temporally correlated noise on the orientation of a magnetic swimmer. Our investigation was motivated by observations that the noise in the orientation of biological magnetic swimmers, magnetotactic bacteria, typically exceeds the expected thermal noise. We found that the correlation time of the noise affects the effective translational diffusion of the swimmer as well as the precision of its orientation with respect to a magnetic field. The latter is often used to define an effective temperature; however, our results show that this temperature in general does not reflect the effective rotational diffusion coefficient. We also note that the internal nonthermal noise considered here is not the only possible contribution to the observed effective temperatures. An additional contribution that results in similar Langevin curves may be due to active directional changes such as 


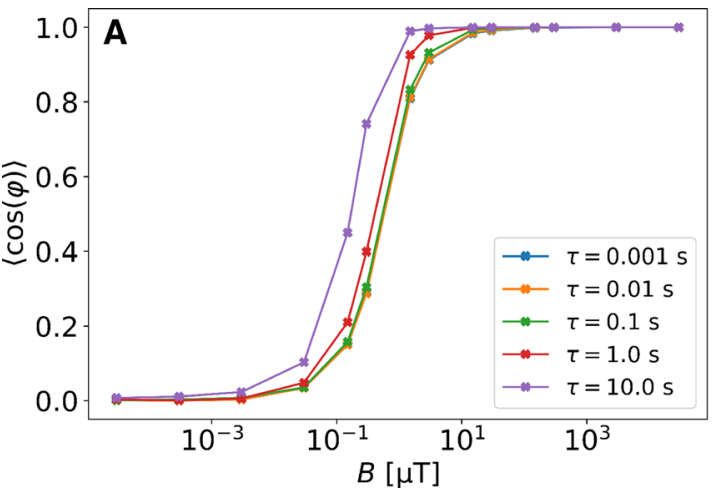

Fig. 3 a Langevin curves and (b) effective temperatures for different correlation times with $D_{c}=0.5 \mathrm{rad}^{2} / \mathrm{s}$. The magnetic strength was set to $m=4.2 \times 10^{-16} \mathrm{Am}^{2}$, corresponding to the average magnetic strength of the magnetotactic bacterium AMB-1 [6]. Trajectories of 1000 swimmers

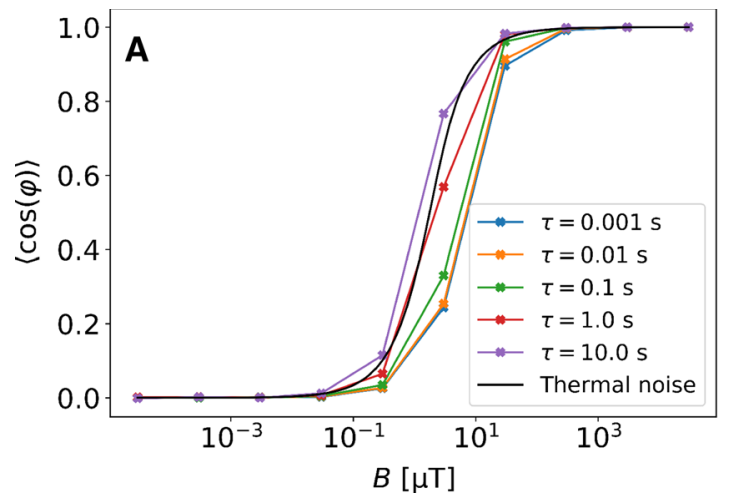

Fig. 4 a Langevin curves for the case of combined thermal and correlated non-thermal noise (colored lines) for $D_{\mathrm{c}}=5 \mathrm{rad}^{2} / \mathrm{s}$ in comparison to thermal noise only at $T=300 \mathrm{~K}$ (black line). The rotational diffusion constant was set to $D_{\mathrm{r}}=1 \mathrm{rad}^{2} / \mathrm{s}$ and the magnetic strength to $m=4.2 \times 10^{-16} \mathrm{Am}^{2}$. Trajectories of 500 swimmers were analyzed. $\mathbf{b}$ Corresponding effective temperatures as obtained from the Langevin curve (crosses) and from rota-

tumbles, flicks and reversals, which should be detected as discrete events in the trajectories [18].

Acknowledgements This work was supported by the DFG (grant number KL818/2-2) within the priority program SPP1726 on microswimmers.

Funding Information Open Access funding enabled and organized by Projekt DEAL.

\section{Author contribution statement}

VT and SK conceived the project. SS, JR and JH performed simulations. SS, VT and SK wrote the paper.

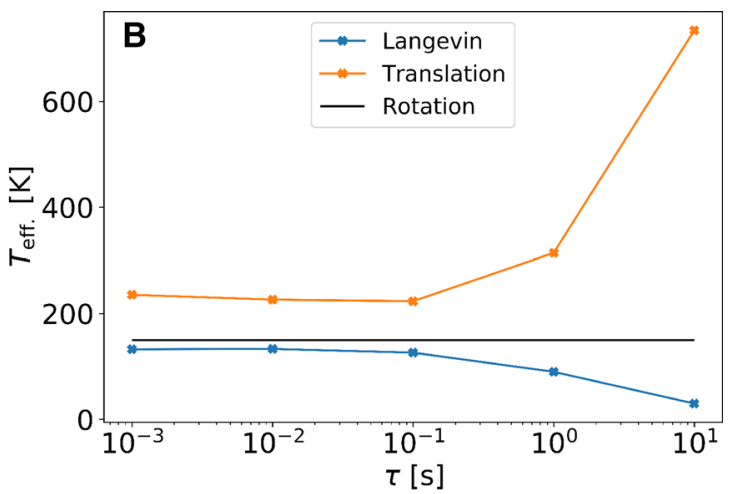

were used for statistical analysis. In addition to the effective temperature obtained from the Langevin curve $\left(T_{\mathrm{eff}}\right)$, we also show effective temperatures derived from rotational and translational diffusion $\left(T_{\mathrm{eff}}^{(\mathrm{r})}\right.$ and $T_{\mathrm{eff}}^{(\mathrm{t})}$, respectively) in (b)

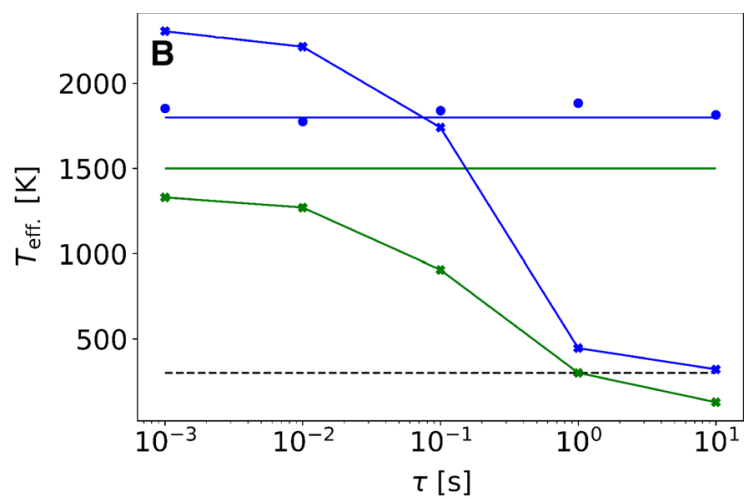

tional diffusion (circles) for the case with both noise terms (blue) and with the correlated noise only (green). The black line indicates the actual temperature of $300 \mathrm{~K}$. For $T_{\mathrm{eff}}^{(\mathrm{r})}$ the simulation data for the case with both noise terms (blue circles) agree with the exact value for the case with only correlated noise (green horizontal line) plus 300K (blue horizontal line)

Open Access This article is licensed under a Creative Commons Attribution 4.0 International License, which permits use, sharing, adaptation, distribution and reproduction in any medium or format, as long as you give appropriate credit to the original author(s) and the source, provide a link to the Creative Commons licence, and indicate if changes were made. The images or other third party material in this article are included in the article's Creative Commons licence, unless indicated otherwise in a credit line to the material. If material is not included in the article's Creative Commons licence and your intended use is not permitted by statutory regulation or exceeds the permitted use, you will need to obtain permission directly from the copyright holder. To view a copy of this licence, visit http://creativecomm ons.org/licenses/by/4.0/. 


\section{References}

1. D. Chandler, Introduction to Modern Statistical Mechanics (Oxford Univ. Press, Oxford, 1987)

2. D. Mizuno, C. Tardin, C.F. Schmidt, F.C. MacKintosh, Science 315, 370 (2007)

3. H. Turlier, D.A. Fedosov, B. Audoly, T. Auth, N.S. Gov, C. Sykes, J.-F. Joanny, G. Gompper, T. Betz, Nat. Phys. 12, $513(2016)$

4. A.J. Kalmijn, IEEE Trans. Magnetics 17, 1113 (1981)

5. C. Rosenblatt, F.F. Torres de Araujo, R.B. Frankel, Biophys. J. 40, 83 (1982)

6. R. Nadkarni, S. Barkley, C. Fradin, PLoS One 8, e82064 (2013)

7. X. Zhu, G. Xin, L. Ning, L.-F. Wu, C. Luo, Q. Ouyang, Y. Tu, G. Chen, Integr. Biol. 6, 706 (2014)

8. J. Elgeti, R.G. Winkler, G. Gompper, Rep. Prog. Phys. 78, $056601(2015)$
9. C. Bechinger, R. Di Leonardo, H. Löwen, C. Reichhard, G. Volpe, G. Volpe, Rev. Mod. Phys. 88, 045006 (2016)

10. S. Klumpp, M. Bennet, C.T. Lefévre, D. Faivre, Phys. Rep. 789, 1 (2019)

11. D.A. Bazylinski, R.B. Frankel, Nat. Rev. Microbiol. 2, $217(2004)$

12. D. Faivre, D. Schuler, Chem. Rev. 108, 4875 (2008)

13. A. Jentzen, P. Kloeden, Milan J. Math. 77, 205 (2009)

14. H. Risken, The Fokker-Planck Equation (Springer, Berlin, 1989)

15. M.A. Berg, H. Kaur, J. Chem. Phys. 146, 054104 (2017)

16. N.G. van Kampen, J. Stat. Phys. 54, 1289 (1989)

17. P. Langevin, J. Phys. Theor. Appl. 4, 678 (1905)

18. A. Codutti, K. Bente, D. Faivre, S. Klumpp, PLOS Comp Biol. 15, e1007548 (2019) 